\title{
Quiste de Tarlov y disfunción vesical sintomática
}

\author{
Ruibal Moldes M, Sánchez Rodríguez-Losada J, López García D, Casas Agudo V, \\ Janeiro País JM, González Martín M.
}

Servicio de Urología. Hospital Juan Canalejo. La Coruña.

Actas Urol Esp. 2008;32(10):1035-1036

\section{RESUMEN}

QUISTE DE TARLOV Y DISFUNCIÓN VESICAL SINTOMÁTICA

El quiste de Tarlov o quiste perineural son lesiones de las raíces nerviosas localizadas a nivel de la región sacra y de etiología incierta. La mayoría de estos quistes permanecen asintomáticos y carecen de relevancia clínica. Los quistes sintomáticos son infrecuentes y los síntomas más habituales son el dolor y las radiculopatías.

Nosotros presentamos el caso de una mujer de 53 años con un quiste de Tarlov sintomático ( síndrome de frecuencia y urgencia miccional) que tras el tratamiento quirúrgico presenta una mejoría clínica importante.

Palabras clave: Quiste Tarlov. Sintomático. Disfunción. Vesical.

\section{ABSTRACT}

TARLOV CYST AND SYMPTOMATIC BLADDER DISFUCTION

Tarlov cysts or perineural cyst are lesions of the nerve roots located at the sacral level and uncertain aetiology. Most of these cysts remain asymptomatic with no clinical relevance. The symptomatic cysts are uncommon and the usual symptoms are pain or radiculopathy.

We report the case of a 53 year old woman with a symptomatic cyst (with a history of frequency and urgency syndrom), that disappears after surgery.

Keywords: Tarlov cyst. Symptomatic. Bladder. Disfuction.

$\mathrm{L}^{\infty}$ os quistes de Tarlov, también conocidos como quistes perineurales, son lesiones de las raíces nerviosas fundamentalmente localizadas a nivel sacro. Aunque su etiología es incierta se han postulado diferentes teorías, siendo los pequeños traumatismos repetidos o el incremento de la presión del líquido cerebroespinal los posibles mecanismos etiológicos más aceptados actualmente ${ }^{1}$. A favor del primero como posible factor desencadenante destaca el hecho de que muchos pacientes relatan antecedentes de traumatismos lumbo-sacros no graves. Muchas de estas lesiones permanecen asintomáticas a lo largo de la vida de los pacientes y generalmente se descubren como hallazgos incidentales en estudios de imagen (TAC o RMN), carentes de relevancia clínica y que no precisan tratamiento.
Los quistes sintomáticos son infrecuentes, con una mayor incidencia en mujeres, siendo el dolor y la radiculopatía los síntomas más habituales.

Presentamos el caso de una paciente de 53 años remitida a nuestro servicio por una historia de un sindrome de urgencia y frecuencia que desapareció tras la resección quirúrgica de dicha lesión.

\section{CASO CLINICO}

Mujer de 53 años de edad sin antecedentes relevantes de interés, salvo un leve traumatismo sacro a los 9 años de edad, que es remitida a nuestras consultas por un cuadro clínico de 7 meses de evolución con molestias en hipogastrio, urgencia y frecuencia miccional que no responde a tratamiento farmacológico, precisando en ese momento la paciente derivados opiáceos y ansiolíticos para mitigar los síntomas. 
La exploración física, incluyendo evaluación ginecológica, así como hemograma, bioquímica, urocultivo y citología de orina fueron normales. Tampoco se encontraron anomalías en la urografía intravenosa ni en el estudio urodinámico. Finalmente una RMN lumbosacra mostró la presencia de una lesión quística perineural extradural de 30×20x20 mm localizada a nivel de la segunda raíz sacra izquierda (S2) (Fig. 1). Una posterior radiculografía mostró una mínima comunicación del quiste con el saco dural.

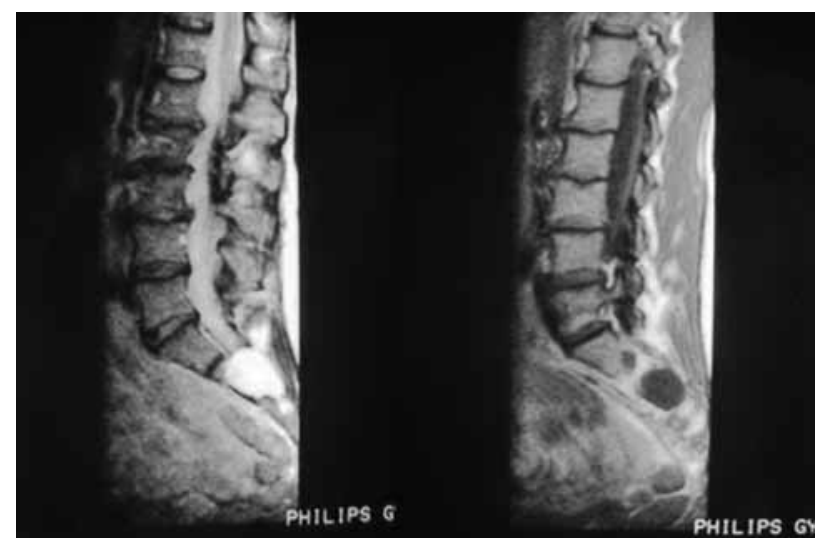

FIGURA 1. RMN de quiste de Tarlov: legión quistica en el canal sacro.

Con el diagnóstico final de un quiste de Tarlov a nivel de S2, la paciente se remite al Servicio de Neurocirugía para intervención quirúrgica. La cirugía consistió en una laminectomía sacra, apertura del quiste, descompresión de la raíz sacra (S2) y recomposición de S3 con duramadre liofilizada y sellado de la cavidad quística con tejido subcutáneo.

A los 5 años de la cirugía la paciente presenta ausencia del dolor y una dramática reducción de su sintomatología urinaria con una importante mejoría en su calidad de vida.

\section{DISCUSIÓN}

Este tipo de lesiones quísticas perineurales fueron descritas inicialmente por Tarlov en 1938 como hallazgos incidentales en autopsias. Desde entonces menos de 100 casos con relevancia clínica han sido publicados en la literatura ${ }^{2}$. Aunque su etiología es incierta, actualmente se han postulado los microtraumatismos a nivel lumbosacro como un posible factor de riesgo. En nuestro caso la paciente relata el antecedente de un traumatismo sacro no grave a los 9 años de edad.

Generalmente se tratan de lesiones asintomáticas carentes de relevancia clínica, descubiertas incidentalmente en estudios radiológicos y por lo tanto que no precisan tratamiento. En los infrecuentes casos con manifestaciones clínicas, los signos y síntomas, así como los hallazgos neurológicos, dependen de cuál o cuáles sean las raíces nerviosas sacras o coccígeas comprometidas por la lesión quística. Así, pueden cursar con dolor, parestesias, hipostesias y trastornos motores, tanto a nivel de extremidades inferiores como áreas genital, perineal o lumbosacra. En nuestro caso el hecho de que sólo existiese un trastorno miccional como única manifestación, lo hace todavía más infrecuente siendo su diagnóstico difícil si no existe una sospecha previa. Sin embargo y a pesar de su baja incidencia, debería considerarse en el diagnóstico diferencial sobre todo en mujeres que presentan dolor lumbosacro o radiculopatía asociados a los sintomas urinarios.

Tanto la TAC como la RMN son excelentes métodos de estudio que permiten detectar masas espinales extradurales. La mielografía lumbosacra seguida de la TAC, permiten diagnosticar este tipo de lesiones y diferenciarlas de otras lesiones espinales ${ }^{3}$.

Así como existe un consenso en cuanto a que las lesiones asintomáticas no precisan tratamiento, es más controvertida la decisión de cuáles son los pacientes con quistes sintomáticos que deberían ser tratados. Aquellos pacientes con lesiones menores a $1,5 \mathrm{~cm}$ y $\sin$ sintomas de radiculopatía apenas experimentan beneficios con el tratamiento quirúrgico. Los mejores resultados se obtienen en aquellos pacientes con lesiones mayores de $1,5 \mathrm{~cm}$ y sintomas radiculares, disfunciones vesicales o intestinales ${ }^{2}$. En nuestro caso se trataba de un quiste de $3 \mathrm{~cm}$ que mejoró de modo espectacular tras la cirugía. La aspiración percutánea no es recomendable no sólo por el escaso beneficio obtenido, sino además por el riesgo de sangrado o infección con el consiguiente empeoramiento de los sintomas. El tratamiento con cirugía abierta constituye el tratamiento de elección.

\section{REFERENCIAS}

1. Strully KJ, Heiser S. Lumbar and sacral cysts of meningeal origin. Radiology. 1954;62(4):544-549.

2. Voyadzis JM, Bhargava P, Henderson FC. Tarlov cysts: a study of 10 cases with review of the literature. J Neurosurgery. 2001;95(1 Suppl): 25-32

3. Nabors MW, Pait TG, Byrd EB, Karim NO, Davis DO, Kobrine AI, et al. Update assessment and current classification of spinal meningeal cysts. J Neurosurgery. 1988;68(3):366-377.

Correspondencia autor: Dr. M. Ruibal Moldes Servicio de Urología. Hospital Juan Canalejo Xubias de Arriba, 84 - 15006 La Coruña. Tel.: 981178000

E-mail autor: ruibalmoldes@terra.es

Información artículo: Nota clínica

Trabajo recibido: marzo 2007

Trabajo aceptado: mayo 2007 